\title{
APRENDIZADO BASEADO EM JOGOS DIGITAIS NO ENSINO DE ANATOMIA UTILIZANDO GAMIFICAÇÃO: UMA REVISÃO SISTEMÁTICA DA LITERATURA
}

\author{
Poliana Francibele de Oliveira Pereira ${ }^{1}$ \\ Patrícia Jantsch Fiuza ${ }^{2}$ \\ Robson Rodrigues Lemos ${ }^{3}$
}

\begin{abstract}
Resumo: Muito tem-se discutido nos últimos anos sobre os efeitos positivos da utilização de jogos digitais como alternativa para o ensino e aprendizagem na área médica, mais especificamente no estudo da anatomia do corpo humano, com estudos focados na visualização de resultados. Partindo de que tanto os Jogos como a gamificação são uma forma de ensinar de forma motivadora, particularmente na medicina essa forma de ensinar tem sido cada vez mais utilizada ultimamente. Por esse motivo propôs-se a realização de um estudo através de uma revisão sistemática da literatura abordando de forma exploratória através de uma análise bibliométrica a variada natureza dos resultados de estudos experimentais destinados a examinar a eficácia dos jogos produzidos direcionados ao ensino de conteúdos de anatomia, utilizando aspectos de gamificação como forma motivacional. Os resultados apresentados neste campo de pesquisa, apesar de serem favoráveis, ainda não são suficientes e expõe uma carência de estudos mais concretos e especialmente, mais prolongados, devido à necessidade de dados mais consistentes.
\end{abstract}

Palavras-chave: Tecnologias na educação; Aprendizagem baseado em Jogos digitais; Gamificação; Anatomia Virtual;

\section{Learning based on digital games in anatomy teaching using gamification: A Systematic Review of Literature}

Astract: Much has been discussed in recent years about the positive effects of using digital games as an alternative to teaching and learning in the medical field, more specifically in the study of the anatomy of the human body, with studies focused on visualizing results. Since both games and gamification are a way of teaching in a motivating way, particularly in medicine, this way of teaching has been increasingly used lately. For this reason a study was proposed through a systematic review of the literature exploring in an exploratory way through a bibliometric analysis the varied nature of the results of experimental studies destined to examine the effectiveness of games produced directed to the teaching of anatomy contents, using aspects of gamification as a motivational form. The results presented in this research area, although favorable, are still not enough and show a lack of more concrete and especially longer studies due to the need for more consistent data.

\footnotetext{
${ }^{1}$ Mestranda em Tecnologias da Informação e Comunicação pela universidade Federal de Santa Catarina UFSC.po.fran@hotmail.com

2 Professora da Universidade Federal de Santa Catarina - UFSC campus Araranguá. patricia.fiuza@ufsc.br

3 Professor da Universidade Federal de Santa Catarina (Campus Araranguá). robson.lemos@ufsc.br
} 
Keywords: Technologies in education; Learning based on digital games; Gamification; Virtual Anatomy;

Os avanços tecnológicos ocorridos nos últimos anos têm contribuído para a melhoria da prestação de serviços em diversas áreas. No contexto educacional, novos sistemas de aprendizagem têm sido utilizados buscando favorecer processos educacionais em ambientes realistas e interativos (Machado et. al, 2011, p. 255). Estudos realizados mostram que aprender com objetos que fornecem alternativas educacionais para 0 raciocínio envolvendo uma situação de resolução de problemas são mais adequados para a aprendizagem por usarem tipos de objetos que reduzem a carga cognitiva da memória de trabalho facilitando assim o processo de aprendizagem (Rondon, 2013, p. 2). Os jogos digitais são exemplos disso, onde os mecanismos permitem uma aprendizagem construtiva, situada e experiencial, reforçada pela experimentação ativa e imersão no jogo (Girard, 2013, p. 207).

Assim como jogos digitais, uma outra forma de estimular o interesse e engajamento do educando é a gamificação, que tem por base a utilização de elementos dos games fora do contexto dos games, com a finalidade de motivar os indivíduos à ação, auxiliar na solução de problemas e promover aprendizagens (Fardo, 2013, p. 1). Mas ao falarmos de motivação, chegamos a uma das categorias de constituição descritas por Moos (2010, p. 267), a orientação de objetivos que se distingue em objetivos de domínio e objetivos de desempenho. De acordo com Pintrich (2000, p. 477) objetivos de domínio refere-se ao desejo de desenvolver habilidades, dominar novos conhecimentos ou novos conjuntos de habilidades, já objetivos de desempenho refere-se ao desejo de demonstrar a capacidade de sucesso, superando os outros enquanto despendem o menor esforço possível. De acordo com Erhel (2013, p. 158), esta pode ser uma distinção particularmente útil no caso dos jogos e gamificação, pois suas dimensões divertidas e educacionais podem induzir os alunos a se concentrarem nos objetivos de desempenho, alcançando uma maior pontuação ou em objetivos de domínio no dominar o conhecimento.

Partindo de que tanto os Jogos como a gamificação são uma forma de ensinar de forma motivadora, a Medicina é uma área que tem se beneficiado ultimamente dessas ferramentas (Machado et. al, 2011, p. 255), e no ensino de áreas como anatomia que 
podem conter conteúdos difíceis e complexos, tornam tais ferramentas importantes aliados. Dentro deste contexto o presente trabalho visa discutir a forma que a literatura tem tratado o aprendizado baseado em jogos digitais bem com recursos de gamificação no ensino de conteúdos na área médica, mais especificamente, o estudo da anatomia do corpo humano através de uma revisão sistemática da literatura.

\section{Ensino de Anatomia do corpo humano}

Vários teóricos, como Paulo Freire (2002, p. 22), já demonstraram que a prática é o grande motivador do aprendizado na educação de adultos, e Martins (2008, p. 24) afirma que um exemplo foi observado na medicina, pelo fato de ser mais interessante e mais produtivo quando o discente confronta-se com problemas em um ambiente mais realista possível, dessa forma, estratégias de ensino que exigem a participação ativa do estudante na busca de soluções para problemas reais, ou construídos com objetivos pedagógicos, têm corroborado para o ensinamento desses.

Como Janssen (2015, p. 2) afirma, na educação médica, os principais temas como a anatomia podem se beneficiar da adoção direcionada de ferramentas de aprendizagem baseadas em tecnologia, como os jogos digitais, pois a anatomia é considerada um assunto desafiador para os estudantes de medicina aprenderem devido à complexidade do sujeito e à amplitude do currículo médico. E, do ponto de vista de Gomes (2016, p. 2) o ensino da Anatomia do Aparelho Locomotor Humano é bastante amplo e constituído pelo estudo dos sistemas esquelético, muscular, articular e planos e eixos do corpo humano, portanto, estudos relacionados a este sugerem o conhecimento aprofundado de peculiaridades, tais como: estruturas e acidentes anatômicos com terminologias bastante complexas, sendo que neste campo existem as fragilidades do material educacional utilizado pelos discentes para aprendizagem da Anatomia Humana.

Por esse motivo a utilização de estratégias educacionais inovadoras como o uso de jogos contendo objetos em 3D poderia facilitar a aprendizagem deste conteúdo, levando os alunos a um ambiente mais realista e com maior facilidade de visualização de objetos que outrora em outros materiais de aprendizagem como livros, ou réplicas anatômicas do corpo humano não daria essa facilidade de visualização.

\section{Aprendizado Baseado em Jogos Digitais e Gamificação}


Os jogos fazem parte da vida de todos os indivíduos desde os tempos mais remotos, estando presentes em várias fases da vida, desde a infância até a vida adulta, e ao longo da história, vem influenciando positivamente no desenvolvimento dos aspectos afetivo, físico, social e moral dos que o praticam (Gomes, 2016, p. 4). Atualmente, os jogos ou aprendizagem baseada em jogos, se tornaram um foco significativo no campo da educação (Van Nuland et. al., 2014, p. 2), sendo considerados excelentes ferramentas instrucionais, na medida em que entretêm, motivam, facilitam o aprendizado, além de aumentar a capacidade de retenção do que foi ensinado.

Já a gamificação deriva diretamente da popularização dos jogos, e de suas capacidades intrínsecas de motivar a ação, resolver problemas e potencializar aprendizagens nas mais diversas áreas do conhecimento e da vida dos indivíduos (Fardo, 2013, p. 2). Diferentemente dos jogos, a gamificação é aplicada de forma ampliada onde pouco percebe-se a sua inclusão no dia a dia. Por sua vez, no contexto de educação, a gamificação compreende a ideia de adicionar elementos, mecânicas e lógica dos jogos para engajar as pessoas com a finalidade a aprendizagem.

Destaca-se que a gamificação aliada a utilização de jogos no ensino pode deixar o ambiente mais agradável e fazer com que $\mathrm{o}$ aluno ao aprender coloque em prática a competição como forma motivadora, relacionando a teoria da Aprendizagem Baseada em Competição (ou Competition Based-Learning - CBL - em inglês) definida por Burguillo (2010, p. 569) como um processo de receber instrução ou adquirir conhecimento dentro de um ambiente competitivo, pois procura incentivar os alunos além da competição num grupo por melhor colocação no ranking, até realizar as atividades para obter objetivos préestabelecidos, como medalhas e prêmios.

\section{BUSCA SISTEMÁTICA NA LITERATURA}

Esta pesquisa pode ser classificada como exploratória utilizando-se de procedimentos de Revisão Sistemática da Literatura (RSL). A partir da RSL foi escolhida a análise bibliométrica para analisar os dados bibliográficos. Para realizar este trabalho, foram desenvolvidas duas etapas: Planejamento e Estratégias de Execução.

Durante o estágio de planejamento foram definidos os níveis de pesquisas, como subseção destes foi definido: a questão de pesquisa, os descritores, os critérios de inclusão e exclusão, e as bases de dados a serem utilizadas. A RSL foi definida como 
necessária para responder à questão de como se dá a evolução dos estudos teóricos e empíricos relacionados à Educação Baseada em jogos Digitais e utilização de conceitos de gamificação na área de ensino de anatomia. Às estratégias de execução dividem-se em duas subseções: etapa da coleta de dados para os estudos, e a seção 2 descreve como foram feitas as representações das análises dos dados.

Como estratégia para execução da busca sistemática na literatura utilizou-se de duas bases de dados: SCOPUS Elsevier e Web of Science (WOS). Sendo que a seleção das bases de dados foi feita com base na representatividade acadêmica que estas têm mundialmente.

Etapa 1: Definição dos termos e critérios de busca. Foram utilizadas as buscas encadeadas nas bases de dados e os seguintes números de documentos foram apresentados conforme os descritores, estipulados na tabela 3.

Tabela 3 - Resultados da busca dos termos nas bases de dados.

\begin{tabular}{c|c|c}
\hline String de Busca & Scopus & WoS \\
\hline Education & 1.699 .186 & $\begin{array}{c}710.79 \\
3\end{array}$ \\
\hline "Digital Game Based learning" OR Gamification & 8.633 & 691 \\
\hline Health OR anatom* & 2.184 & 64 \\
\hline
\end{tabular}

Fonte: Elaborado pelos Autores (2017).

Etapa 2: Limitação da busca de artigos completos. Esse critério foi aplicado para poder compreender melhor os objetivos, conceitos, temas e conclusões dos documentos. Com isso na Scopus a busca resultou em 990 publicações e na WoS sobraram 29 publicações.

Etapa 3: Limitação da busca para publicações que utilizam apenas palavras chaves que fazem parte do escopo estabelecido. Com isso na Scopus restaram 322 publicações e na WoS, 31.

Etapa 4: Avaliação crítica dos estudos encontrados. Para os documentos restantes da etapa anterior foram estipulados critérios de exclusão para a análise, apresentados na Tabela 4.

Tabela 4 - Critérios de Busca e Exclusão. 


\begin{tabular}{l|cc}
\multicolumn{1}{c|}{ Critério de Exclusão } & Scopus & WOS \\
\hline $\begin{array}{l}\text { Título, resumo e palavras-chaves não relacionados aos descritores } \\
\text { pesquisados }\end{array}$ & 176 \\
Artigos repetidos nas bases de dados & 12 & - \\
Artigos pagos & 34 & 6 \\
$\begin{array}{l}\text { Após a leitura do resumo identificado que assunto não relaciona com } \\
\text { descritores }\end{array}$ & 57 & 7 \\
$\begin{array}{l}\text { Após a leitura do documento identificado que o assunto não relaciona com } \\
\text { pesquisa }\end{array}$ & 28 \\
\hline $\begin{array}{l}\text { Documentos restantes do processo de seleção para análise estudos teórico- } \\
\text { empíricos }\end{array}$ & $\mathbf{1 5}$ \\
\hline
\end{tabular}

Fonte: Elaborado pelos Autores (2017).

Etapa 5: Análise dos documentos restantes. Os 18 documentos restantes foram analisados e categorizados por variáveis tratadas nas publicações para aprofundar o conhecimento dos estudos.

\section{RESULTADOS}

Após a busca sistemática da literatura chegou-se a 18 trabalhos selecionados, que foram escritos por 70 autores vinculados a 32 instituições de 12 países e publicados em 12 periódicos. Nesta analise obteve-se documentos relevantes a partir de 2010, porém percebe-se que o tema em estudo recebeu uma grande atenção em 2015 com 8 documentos abordando o assunto, representando mais de $40 \%$ dos documentos selecionado, conforme mostra o gráfico 1.

Gráfico 1 - Frequência das publicações por ano no período (2010-2016).

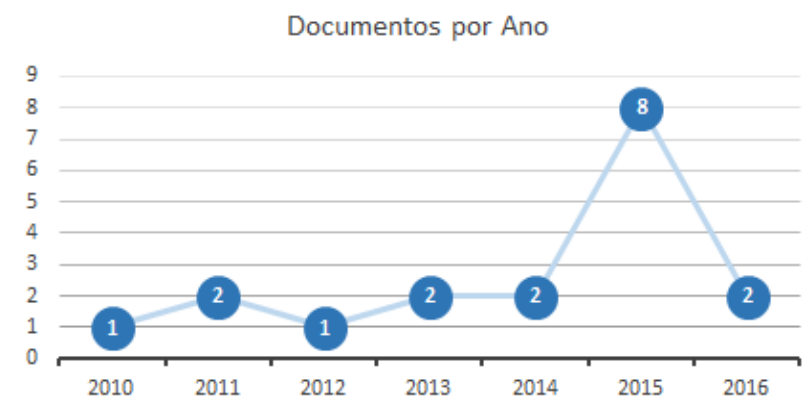

Fonte: Elaborado pelos Autores (2017).

Outro fator analisado foram os países de origem das publicações, os Estados Unidos lideram a lista com 6 artigos publicados, seguido pelo Canadá com 5 artigos, estes 
são responsáveis juntos por $61 \%$ das publicações sobre o tema, contra $39 \%$ dos documentos distribuídos entre 10 países.

$\mathrm{Na}$ tabela 5 procurou-se enfatizar os tipos de pesquisas abordadas pelos autores em cada documento com a finalidade de saber em que situação foram desenvolvidos novos softwares e em que momento foram adaptados softwares para a educação da Anatomia do corpo humano. Além disso, foi analisado o tipo de estudo apresentados que foram subdivididos em 4 níveis:

- EC1: Estudos comparativos em grupos com a utilização de software e método tradicional.

- EC2: Estudos comparativos em grupos com e sem conhecimento prévio, grupos com conteúdos diferentes no software ou grupos de níveis de escolaridades diferentes.

- EC3: Estudos em grupos entre mais de uma abordagem ou mais de um tipo de jogo.

- O: Outro.

E por fim, foi avaliado o tipo de análise que cada documento apresentou na finalização dos estudos, para analisar o objetivo de cada documento. A tabela 5 apresenta esses resultados.

Tabela 5: Tipo de Pesquisa, estudo e análise

\begin{tabular}{lllcc}
\hline Ano & Autor & Tipo de Pesquisa & Tipo de Estudo & Tipo de análise \\
\hline 2015 & Albu, M., Atack, L. & Aplicação de um SW & EC1 & Motivação \\
\hline 2015 & Cain, J., Piascik, P. & $\begin{array}{l}\text { Aplicação de } \\
\text { gamificação }\end{array}$ & EC3 & Análise de desempenho \\
\hline 2010 & Cowan, B., et. al & $\begin{array}{l}\text { Desenvolvimento de } \\
\text { SW }\end{array}$ & O & Usabilidade \\
\hline 2015 & Day-Black, C.,et al. & Aplicação de um SW & EC3 & Aprendizagem \\
\hline 2016 & De-Marcos, L et. al. & Análise comparativa & EC3 & Análise de desempenho \\
\hline 2013 & Erhel, S., Jamet, E. & Aplicação de um SW & EC2 & Análise de desempenho e \\
motivação
\end{tabular}


Revista do Programa de Pós-Graduação em Educação - UNESC

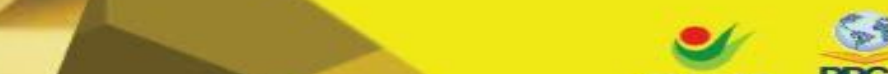

unesc PPG

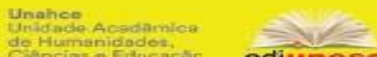

\begin{tabular}{|c|c|c|c|c|}
\hline 2012 & Hannig, A., et. al. & $\begin{array}{l}\text { Desenvolvimento de } \\
\text { SW }\end{array}$ & EC2 & $\begin{array}{c}\text { Análise de desempenho e } \\
\text { usabilidade }\end{array}$ \\
\hline 2015 & Janssen, A., et. al. & $\begin{array}{l}\text { Desenvolvimento de } \\
\text { SW }\end{array}$ & EC2 & Aprendizagem e motivação \\
\hline 2011 & $\begin{array}{l}\text { Kanthan, R., Senger, J.- } \\
\text { L. }\end{array}$ & $\begin{array}{l}\text { Desenvolvimento de } \\
\text { SW }\end{array}$ & EC2 & $\begin{array}{l}\text { Análise de desempenho e } \\
\text { motivação }\end{array}$ \\
\hline 2016 & Mawhirter, D. A., & Aplicação de um SW & EC3 & Aprendizagem \\
\hline 2014 & Nevin, C.R., et. al. & $\begin{array}{l}\text { Desenvolvimento de } \\
\text { SW }\end{array}$ & EC2 & Aprendizagem e usabilidade \\
\hline 2015 & Nicolaidou, I., et.al & $\begin{array}{l}\text { Desenvolvimento de } \\
\text { SW }\end{array}$ & $\mathrm{EC} 2$ & Aprendizagem \\
\hline 2011 & Reilly, F.D. & Análise comparativa & EC3 & $\begin{array}{l}\text { Análise de desempenho e } \\
\text { motivação }\end{array}$ \\
\hline 2013 & Rondon, S., et al. & Aplicação de um SW & EC1 & Análise de desempenho \\
\hline 2015 & $\begin{array}{l}\text { Sung, H.-Y., Hwang, G.- } \\
\text { J. }\end{array}$ & $\begin{array}{l}\text { Desenvolvimento de } \\
\text { SW }\end{array}$ & EC1 & Aprendizagem e motivação \\
\hline 2015 & Van Nuland, S.E. & $\begin{array}{l}\text { Aplicação de } \\
\text { gamificação }\end{array}$ & EC1 & Aprendizagem e motivação \\
\hline
\end{tabular}

Fonte: Elaborado pelos Autores (2017).

Os resultados quanto ao tipo de pesquisa, destacam que em $50 \%$ dos trabalhos foram desenvolvidos novos softwares, mostrando assim, o grande crescimento de novos meios de aprendizagem na anatomia humana como no caso a utilização de jogos. Outros casos $(27,7 \%)$ foram de aplicação de softwares adaptados para o ensino de anatomia. Também foram apresentados 2 casos da aplicação de gamificação em grupos de alunos e 2 análises comparativas, que avaliam o desempenho de alunos com a utilização de mais de um tipo de abordagem.

No quesito tipos de estudos, foi abordado $27,7 \%$ do tipo EC1, envolvendo a aplicação de um determinado conteúdo utilizando um software em contrapartida ao estudo tradicional. Já 38,9\% apresentaram o tipo de estudo EC2, que procurou avaliar o aluno com e sem conhecimento prévio do assunto, com o mesmo software, além de casos de método comparativo entre escolaridade de estudantes, e comparação de aprendizagem em turmas diferentes. Na categoria EC3 com artigos com a aplicação de mais de uma abordagem teve $27,7 \%$ documentos, e caso de comparação entre mais de um modelo de jogo. $\mathrm{O}$ (Outro) foi um documento que analisou a usabilidade de um jogo para anatomia do corpo humano (Cowan, 2010). Os estudos comparativos mostram que 
os autores procuram avaliar a melhor metodologia ou estratégia de avaliação, levando em consideração aplicação de estudos com grupo de controles, a fim de ver o que se adapta melhor ao seu estudo.

Os resultados de tipo de análise são bem semelhantes, sendo que a maioria dos documentos procurou avaliar nível de desempenho dos alunos, ou avaliação de conhecimento, usando métodos estatísticos. Outro fator apresentado em grande parte dos documentos em conjunto com nível de desempenho, foi a avaliação da motivação dos usuários ao utilizarem um software de estudo sobre anatomia do corpo humano. E por fim alguns apresentam o termo análise de usabilidade, procurando avaliar se o software apresentou uma boa interface de usuário.

Gráfico 2: Plataformas utilizadas

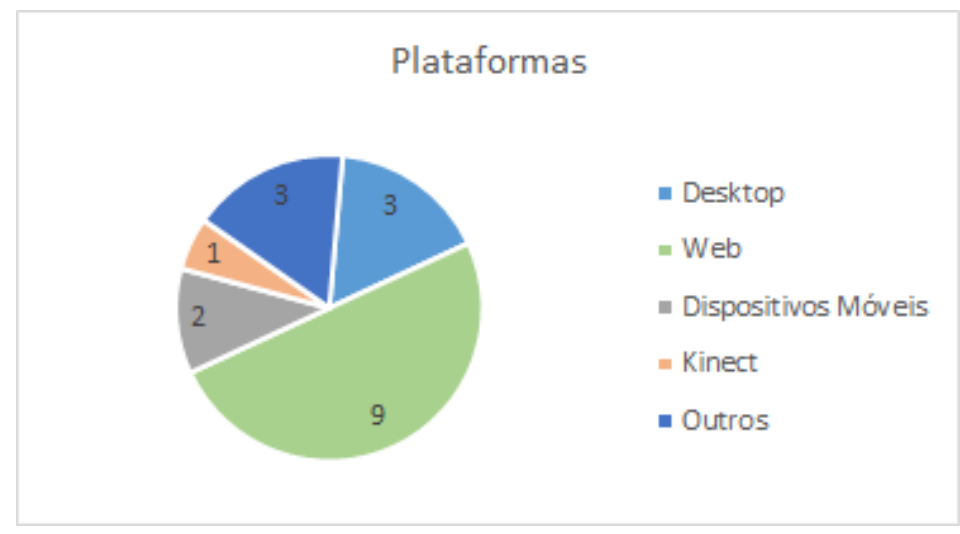

Fonte: Elaborado pelos Autores (2017).

As plataformas mais utilizadas conforme mostram no gráfico 2 é a Web, com $50 \%$ documentos, isso pelo fato de abranger um maior número de usuários, não estando restrito a um ambiente local, seguida por Desktop e também outros, constituído por documentos que analisam estudos sobre os efeitos e benefícios da utilização de gamificação ou DGBL, os artigos enquadrados nesta categoria mostraram resultados favoráveis obtidos com a utilização destes elementos.

$\mathrm{Na}$ sequência, foi analisado os tipos de abordagens apresentadas em cada documento com o intuito de observar quais são as abordagens mais utilizadas pelos autores em seus documentos nos anos analisados, e os resultados estão representados no gráfico 4. 


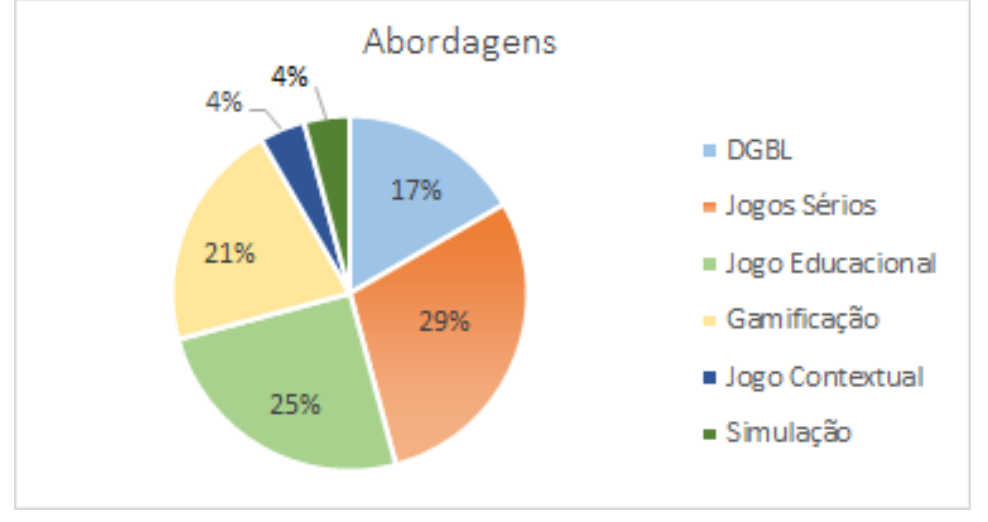

Fonte: Elaborado pelos Autores (2017).

Percebe-se que as abordagens estudadas pelos autores são muito variadas, pois cada um define o que mais se encaixa no seu estudo, e em muitos casos os autores têm utilizado mais de uma abordagem.

\section{CONSIDERAÇÕES FINAIS}

Por meio deste estudo foi possível realizar uma RSL sobre a utilização de jogos e conceitos de gamificação no processo de aprendizagem no ensino de anatomia. Iniciouse com um mapeamento quantitativo das publicações científicas. Constatou-se que o tema tem sido muito investigado, e tem despertado maior atenção da academia a partir de 2011 , onde cerca de $90 \%$ das publicações estão concentradas nos últimos 5 anos. Notase uma grande concentração de estudos provenientes de pesquisadores dos Estados Unidos e Canadá, sendo este último concentrando o maior número de Instituições que publicam sobre $\mathrm{o}$ assunto.

$\mathrm{Na}$ grande maioria dos artigos analisados foram detectados estudos comparativos e avaliação de desempenho, dando assim um peso maior para a pesquisa, que consegue através de métodos estatísticos avaliar se 0 aluno obteve realmente um melhor desempenho com aquele método de ensino. Além disso, os tipos de abordagens demonstram como é amplo a utilização de jogos na educação, ou seja, o ensino de modo alternativo e que desperte o interesse do aluno no aprender. Outro aspecto relevante foi no fato de que ao realizar as buscas com os descritores, muitos artigos retornados falavam de estudos sobre resultados da utilização de jogos de anatomia nos próprios pacientes e não como é o foco desta pesquisa, o ensino de estudantes de anatomia, isso nos mostra que a preocupação vai além do ensino de alunos, mas também de pacientes. 
Em termo de resultados quanto à efetividade dos benefícios da utilização de jogos e gamificação no processo de ensino e aprendizagem, é necessária uma investigação mais aprofundada para avaliar os benefícios específicos e efeitos a longo prazo, mas este estudo oferece suporte preliminar para a alegação de que o aprendizado utilizando jogos como forma de ensinar pode melhorar o aprendizado profundo nesta configuração, aumentado o interesse do aluno no que diz respeito ao estudo de anatomia.

\section{REFERÊNCIAS}

ALBU, M.; ATACK, L.; SRIVASTAVA, I.. Simulation and gaming to promote health education: results of a usability test. Health Education Journal, v. 74, n. 2, p. 244-254, 2015.

ARNAB, S. et. al. The development approach of a pedagogically-driven serious game to support Relationship and Sex Education (RSE) within a classroom setting. Computers \& Education, 69, 15-30, 2013.

BURGUILLO, J. C. Using game theory and competition-based learning to stimulate student motivation and performance. Computers \& Education, v. 55, n. 2, p. 566-575, 2010.

CAIN, J.; PIASCIK, P. Are serious games a good strategy for pharmacy education?. American journal of pharmaceutical education? v. 79, n. 4, p. 47, 2015.

COWAN, B. et. al. A serious game for total knee arthroplasty procedure, education and training. Journal of CyberTherapy \& Rehabilitation (JCR), v. 3, n. 3, 2010.

DAY-BLACK, C. Gamification: An Innovative Teaching-Learning Strategy for the Digital Nursing Students in a Community Health Nursing Course. ABNF Journal, v. 26, n. 4, 2015.

DE-MARCOS, L.; GARCIA-LOPEZ, E.; GARCIA-CABOT, A.; On the effectiveness of game-like and social approaches in learning: Comparing educational gaming, gamification \& social networking. Computers \& Education, v. 95, p. 99-113, 2016.

ERHEL, S.; JAMET, E. Digital game-based learning: Impact of instructions and feedback on motivation and learning effectiveness. Computers \& Education, 67, 2013, 156-167.

FARDO, M. L. A gamificação aplicada em ambientes de aprendizagem. RENOTE, 11(1) p. 1 - 9. 2013.

FREIRE, P. Pedagogia da autonomia: saberes necessários à prática educativa. 25. ed. São Paulo: Paz e Terra, p. 1 -54. 2002.

GRAAFLAND, M. et. al. A serious game can be a valid method to train clinical decisionmaking in surgery. World journal of surgery, v. 38, n. 12, p. 3056-3062, 2014. 
GAUTHIER, A.; CORRIN, M.; JENKINSON, J. Exploring the influence of game design on learning and voluntary use in an online vascular anatomy study aid. Computers \& Education, v. 87, p. 24-34, 2015.

GIRARD, C.; ECALLE, J.; MAGNAN, A. Serious games as new educational tools: how effective are they? A meta-analysis of recent studies. Journal of Computer Assisted Learning, v. 29, n. 3, p. 207-219, 2013.

GOMES, V. X. S. S.; MARINHO, A. M. C. P.; CARNEIRO, C. Jogo digital como estratégia para aprendizagem da anatomia do aparelho locomotor humano na perspectiva de discentes do curso de educação física. Revista Tecnologias na Educação. Ano 8.Número/Vol.17. p. 1 - 11. 2016.

HANNIG, A. et al. eMedOffice: A web-based collaborative serious game for teaching optimal design of a medical practice. BMC medical education, v. 12, n. 1, p. 104, 2012.

JANSSEN, A. et al. A little healthy competition: using mixed methods to pilot a team-based digital game for boosting medical student engagement with anatomy and histology content. BMC medical education, v. 15, n. 1, p. 1-10, 2015.

KANTHAN, R., SENGER, J.-L. The impact of specially designed digital games-based learning in undergraduate pathology and medical education. (2011) Archives of Pathology and Laboratory Medicine, 135(1), pp. 135-142.

MACHADO, L. D. S. et. al. Serious games baseados em realidade virtual para educação médica. Revista Brasileira de Educação Médica, 2011, 35(2), 254-262.

MARTINS, M. A. Novas Tendências do Ensino Médico. Revista Associação Médica. Gazeta Médica da Bahia . Brazil. 2008;78 (Suplemento 1):22-24.

MOOS, D. C.; MARROQUIN, E. Multimedia, hypermedia, and hypertext: Motivation considered and reconsidered. Computers in Human Behavior, 26(3), 265-276, 2010.

NEVIN, C. R. et al. Gamification as a tool for enhancing graduate medical education. Postgraduate medical journal, Postgraduate medical journal, postgradmedj, p. 1-9. 2013.

NICOLAIDOU, I. et al. A virtual emergency telemedicine serious game in medical training: a quantitative, professional feedback-informed evaluation study. Journal of medical Internet research, v. 17, n. 6, 2015.

PINTRICH, P. The role of goal orientation in self-regulated learning. In M. Boekaerts, P. Pintrich, \& M. Zeidner (Eds.), Handbook of self-regulation. San Diego, CA. p. 452 - 529. 2000.

REILLY, F. D.; Outcomes from building system courseware for teaching and testing in a discipline-based human structure curriculum. Anatomical sciences education, v. 4, n. 4, p. 190-194, 2011. 


\section{CRIAR EDUCAÇÃO}

Revista do Programa de Pós-Graduação em Educação - UNESC

RONDON, S.; SASSI, F. C.; DE ANDRADE, C. R. F. Computer game-based and traditional learning method: a comparison regarding students' knowledge retention. BMC medical education, v. 13, n. 1, p. 30, 2013.

SUNG, H.; HWANG, G.; YEN, Y.; Development of a contextual decision-making game for improving students' learning performance in a health education course. Computers \& Education, v. 82, p. 179-190, 2015.

VAN NULAND, S. E. et al. Head to head: The role of academic competition in undergraduate anatomical education. Anatomical sciences education, Anatomical Sciences Education. p.1-9, 2014.

Recebido em dezembro 2018

Aprovado em março 2019 